\title{
Tumor mutational burden and immune signatures interplay in renal cell carcinoma
}

\author{
Evgeny Yakirevich, Nimesh R. Patel \\ Department of Pathology, Rhode Island Hospital and Alpert Medical School at Brown University, Providence, RI, USA \\ Correspondence to: Evgeny Yakirevich, MD, DSc. Department of Pathology, Rhode Island Hospital, 593 Eddy Street, APC12, Providence, RI 02903, \\ USA. Email: eyakirevich@lifespan.org. \\ Provenance and Peer Review: This article was commissioned by the Editorial Office, Annals of Translational Medicine. The article did not undergo \\ external peer review. \\ Comment on: Zhang C, Li Z, Qi F, et al. Exploration of the relationships between tumor mutation burden with immune infiltrates in clear cell renal \\ cell carcinoma. Ann Transl Med 2019;7:648.
}

Submitted Feb 05, 2020. Accepted for publication Feb 28, 2020.

doi: $10.21037 /$ atm.2020.02.81

View this article at: http://dx.doi.org/10.21037/atm.2020.02.81

Cancer treatment has been revolutionized by characterizing the genomic landscape of tumors and developing targeted therapies through The Cancer Genome Atlas (TCGA) and comprehensive genomic profiling (CGP). Therapeutic antibodies against immune checkpoint inhibitors (ICPIs) cytotoxic T-lymphocyte-associated protein (CTLA-4), programmed death cell protein $1(\mathrm{PD}-1)$ and programmed death-ligand 1 (PD-L1) show considerable promise in a variety of malignancies, including renal cancer (1); however, durable clinical benefit is rarely achieved. Therefore, new avenues to guide immunotherapy-based treatments are a critical but currently unmet need. The relationship between tumor genotype and the biology of the immune microenvironment driving responses to ICPIs is not completely understood. A key challenge is the identification of tumor intrinsic/tumor microenvironment (TME) characteristics that can predict treatment response. Tumor mutational burden (TMB) is one such emerging approach in many cancers, including renal cell carcinoma (RCC). Extensive immunogenomic analysis of 336 clear cell RCC (CCRCC) patients from TCGA dataset reported by Zhang et al. in a previous issue of the Annals of Translational Medicine provides additional evidence of the relationship between TMB and immune infiltrates in CCRCC (2).

\section{TMB and tumor neoantigens in RCC}

The prevalence of somatic mutations, which include nucleotide substitutions and small insertions/deletions, is highly variable between and within cancer types, ranging from 0.001 per megabase $(\mathrm{Mb})$ to more than 400 per Mb (3). On one side of the spectrum are tumors with low TMB, such as prostate and pancreatic cancer, and certain childhood tumors. On the other side, are tumors with high $\mathrm{TMB}$, including genomically unstable tumors related to chronic mutagenic exposure, such as non-small cell lung carcinoma (NSCLC) and melanoma, or mismatch repair deficient tumors, such as microsatellite unstable (MSI) colorectal and noncolorectal cancer. Certain mutational signatures are associated with the age of the patient at cancer diagnosis; in general, TMB increases with patient age, showing a 2.4-fold difference between age 10 and 90 years (4).

RCC is a tumor with a relatively low TMB, with a median of 1.1 mutations per $\mathrm{Mb}(3,5)$. Within the main RCC subtypes, chromophobe RCC carries the lowest TMB ( $<1$ mutation per $\mathrm{Mb}$ ), while CCRCC and papillary RCC exhibit a similar range of TMB (3). As compared to more hypermutated cancers, even the most heavily mutated RCC shows a relatively low TMB. Within each tumor type, including RCC, there is a substantial subset of cases with high TMB (4).

TMB is most commonly estimated based on next generation sequencing (NGS) of formalin-fixed paraffinembedded (FFPE) tissue samples using whole genome sequencing (WGS), whole exome sequencing (WES), and various CGP targeted gene panels. Although there is currently a lack of standardization, TMB is often calculated 
as the total number of single nucleotide variants (SNVs) and small insertions and deletions (indels) per one million bases $(\mathrm{Mb})$ in a tumor based on NGS data. As with many studies based on WES data, Zhang et al. classified the CCRCC samples from TCGA dataset into low- and highTMB groups according to median cutoff (2). In the clinical setting, CGP with targeted panels has proved to be suitable for TMB estimation. Chalmers et al. have demonstrated that a CGP assay targeting approximately $1.1 \mathrm{Mb}$ of coding genome can accurately assess TMB compared with WES and used 20 mutations/Mb as a cutoff for highTMB (4). However, there is also lack of standardization for differentiating high- from low-TMB.

The vast majority of mutations in solid tumors included for calculation of TMB are SNVs, while indels account for approximately $4 \%$ in the TCGA pan-cancer cohort (6). Currently, there is no consensus as to whether gene fusions should be included in the calculation of TMB. Chalmers et al. exclude recurrent mutations occurring in tumors and truncating mutations of tumor suppressor genes from their TMB calculation to avoid overestimation of TMB, since gene panels are usually heavily targeted at recurrently mutated genomic regions (4).

Zhang et al. have shown that SNVs occur more frequently than insertions or deletions in CCRCC, with $\mathrm{C}>\mathrm{T}$ being the most common substitution (2). Based on the predominance of $\mathrm{C}>\mathrm{T}$ substitutions, CCRCC are classified as signatures $1 \mathrm{~B}$ and 6 tumors, with $1 \mathrm{~B}$ observed in the majority (19 of 30) of cancer types (3). In another report, analysis of TCGA dataset demonstrates that in sharp contrast to other solid tumors, RCCs harbor the highest indel rate as a proportion of their total TMB and the highest overall number of indels, more than double the median proportion of indel mutations in all other cancer types (6). This unique feature is characteristic of clear cell RCC, papillary RCC, and chromophobe RCC, suggesting a possible tissue-specific mutational process contributing to the acquisition of indels in renal cancers. Indels may cause a frameshift, creating a novel open reading frame and producing a large amount of highly immunogenic neoantigenic peptides. Compared with SNV mutations, indels can generate three times more highbinding affinity neoantigens and nine times more mutantspecific binders. Immune gene expression analyses in the RCC cohort have revealed that the presence of mutantspecific neoantigens is associated with upregulation of antigen presenting genes, which strongly correlates with T-cell activation as measured by CD8-positive expression (6). However, more recent analysis has failed to confirm a higher frequency of indels in a larger randomized cohort of advanced metastatic RCC treated with ICPIs (7). Therefore, additional mechanisms of immunogenicity in RCC warrant further investigation. Among them are neoantigens from endogenous retroviruses (ERVs) integrated into the genome, which may be reactivated in tumors and contribute to anti-tumor immunity (8). A human ERV type E was found to be selectively expressed in most cases of CCRCC $(8,9)$. Occasional cases of RCC harbor human papilloma virus (HPV) and hepatitis B virus (HBV) sequences $(8,10)$. Although viral antigens provide new targets for T-cells, they do not contribute to high TMB status. Another potential source of tumor antigens is a unique set of genes, known as cancer testis (CT) antigens, which are not expressed in healthy tissues, except germ cells, but are aberrantly expressed in tumors and are associated with antigen-specific responses in patients harboring these tumors. One of these CT antigens, CSAG2, may be involved in anti-tumor immunity in CCRCC (8).

\section{Predictive and prognostic role of TMB in RCC}

Across different tumor types, tumors with high TMB, such as melanoma, non-small cell lung cancer (NSCLC), and urothelial cancer, are associated with improved response to ICPIs (11-13). In contrast, less-mutated tumor types, such as pancreatic and prostate cancer, are not responsive to immune blockade and are not considered as candidates for immunotherapy. Mechanistically, this may be explained by formation of larger number of neoantigens in tumors with higher $\mathrm{TMB}$, which elicit $\mathrm{T}$ cell immunoreactivity and sensitivity to ICPIs (14). The latter are referred to as "hot tumors" (15). RCC is breaking the rule, being a tumor with a relatively low TMB, approximately ten times lower than melanoma, but manifesting response to ICPIs similarly to melanoma. This is why there are now 3 FDAapproved combination therapies with ICPIs in metastatic kidney cancer (1). One plausible hypothesis is that despite low overall TMB in RCC, frequent indel mutations cause a frameshift to create a large amount of neoantigenic peptides, which are more potent than those produced by the same number of SNVs (6). In patients with melanoma treated with ICPIs, frameshift indel burden appears to be a better predictor of response as compared with SNVs (6). Additional studies are needed in order to directly investigate the relationship between the indel mutation load and response to ICPIs in RCC, as indel mutation burden may be a potential biomarker of response to checkpoint blockade. 
Genomic data from the large cohort of patients with advanced cancers of different types treated with ICPIs have demonstrated the continuous association between higher TMB and superior overall survival (OS) (12). However, the trend of improved survival was observed only in RCC when high TMB was defined as the top $20 \%$ (cutoff 5.9 mutations/Mb in RCC). Decreasing the cutoff to the top 30\% (4.9 mutations/Mb) shifted hazard ratio to shorter OS. Similarly, Zhang et al. have shown that high TMB correlates with poor survival in TCGA patients who were not treated with ICPIs, since high TMB occurs in high-grade advanced stage tumors (2). When another cohort of metastatic RCC patients who did not receive ICPIs was analyzed, no prognostic benefit was observed in the high TMB group (12). In a phase II clinical trial of atezolizumab (anti-PD-L1) in patients with metastatic RCC, TMB and neoantigen burden were not associated with progression free survival (7). Therefore, higher TMB does not appear to improve OS or predict benefit from ICPIs in patients with metastatic RCC.

\section{Immune signatures of RCC}

RCC, along with other tumors, such as melanoma, NSCLC, and head and neck carcinoma, contain high levels of tumor infiltrating lymphocytes and are therefore considered immunologically hot tumors, which are more likely to trigger a strong immune response (15). Based on the analysis of a T-cell-inflamed gene expression signature, CCRCC was found to be the most T-cell inflamed cancer across 31 solid tumor types from the TCGA dataset (16). Immune cytolytic activity as defined by transcript levels of 2 key cytotoxic effectors of activated CD8+ T-cells, granzyme A and perforin, was the highest in CCRCC among 18 solid tumor types (8).

Historically, immune infiltrates in RCC were evaluated by immunohistochemical or flow cytometry analyses of the tumor tissues. The type (CD8+ cells), density, and location (intratumoral versus peritumoral) are considered critical factors in the assessment of immune infiltrates and in the determination of their prognostic impact. The TME in RCC is different from that of other tumor types. In most cancers, the increased number of cytotoxic CD8+ tumor-infiltrating lymphocytes is associated with improved prognosis (17). Paradoxically, both intratumoral and peritumoral CD8+ T-cell density in RCC is associated with poor outcome, possibly due to a positive association between the number of lymphocytes and tumor grade
$(18,19)$. High expression of B-cell signatures and decreased B-cell receptor (BCR) diversity predicts poor survival as well (20).

While immunohistochemistry or flow cytometry suffer from limitations in phenotypic markers, computational methods for quantifying cell fractions from bulk tissue gene expression profiles can accurately estimate the immune composition of tumor tissue. Zhang et al. utilized CIBERSORT, a newly developed in silico algorithm for estimation of specific cell types in a mixed cell population using RNA-seq or microarray gene expression data (21). In contrast to previous studies $(18,19)$, lower levels of CD8+ $\mathrm{T}$ cells and macrophages correlated with poor survival outcomes in CCRCC in the Zhang et al. study (2). These differences may be explained in part by transcriptomic analysis of the entire tumor sample versus protein expression analysis within different tumor compartments (intratumoral, peritumoral).

\section{TMB and immune signatures in RCC}

Data provided by Zhang et al. indicate that TMB may be closely related with the TME. Based on the expression levels of immune-related genes and gene sets, Zhang et al. compared 22 immune fractions between low- and high-TMB groups. Paradoxically, higher mutation rates in RCC are associated with immunologically cold (nonT-cell inflamed) phenotype. The infiltration levels of CD8+ T cells, CD4+ memory resting T cells, M1 and M2 macrophages, as well as dendritic resting cells in the highTMB group were lower compared with that in the lowTMB group. In addition, the high-TMB group showed lower levels of immune genes mainly involved in neutrophil mediated immunity (2).

In another study of 32 cancer types from TCGA dataset, Wang and Li compared expression levels of immunerelated genes and gene-sets between high- and low-TMB groups in each cancer type using a different arbitrary cutoff for high TMB (scores higher than the third quartile value) (22). They found that association between TMB and infiltration densities of 16 different immune cell subpopulations was cancer-type dependent. In 12 cancer types, including CCRCC, expression levels of the immune cell subpopulation gene set were significantly higher in the lower-TMB group, as opposed to one cancer type in which expression levels of this gene-set were significantly higher in the higher-TMB group (22). The expression levels of the tumor-infiltrating lymphocytes (TILs) gene-set were 
significantly higher in the higher-TMB group in 9 cancer types, including CCRCC, while significantly higher in the lower-TMB group of 2 cancer types. Moreover, expression levels of immune checkpoint, cytokine and cytokine receptors, and pro-inflammatory genes were significantly higher in the lower-TMB group as opposed to higherTMB group in CCRCC and several other cancer types (22). Therefore, the association between TMB and the immune signature is cancer-type dependent. In CCRCC and several other cancer types, high TMB appears to inhibit immune cell infiltration, creating immunologically cold phenotype, consistent with the findings of Zhang et al. (2). However, in a group of CCRCC with low TMB, an upregulated immune signature can potentially mediate anti-tumor immune response even with a limited number of neoantigens. One possible therapeutic strategy for high-TMB tumors that are immunologically cold may be improving ICPI blockage by promotion of intratumoral T-cell infiltration via oncolytic virotherapy (23).

The mechanisms governing exclusion of T-cells from TME are under intense study. Tumor-intrinsic WNT/ $\beta$-catenin signalling mediates immune exclusion in melanoma and across other human cancers with high TMB (16). Among 31 tumor types, differences in percentages of $\mathrm{Wnt} / \beta$-catenin-activated patients between immunologically cold and hot tumors were most striking in CCRCC and reached $81 \%$ (16). MAPK signaling is another pathway associated with cancer cell immune evasion. In triple-negative breast cancer, potential activating mutations of MAPK signaling have been correlated with lower density of TILs in residual disease following neoadjuvant therapy (24). As reported by Zhang et al., the higher TMB RCC group correlated with $W n t / \beta$-catenin and MAPK signaling, supporting this mechanism of immune exclusion in high-TMB RCC group. These data provide a strong rationale for the development of pharmacologic inhibitors of these pathways with the aim of restoring immune cell infiltration and augmenting immunotherapy.

\section{Conclusions and future perspectives}

There is accumulating evidence that higher TMB in RCC is associated with immune cell exclusion and creation of an immunologically cold phenotype. Therefore, the search for predictive biomarkers for immunotherapy may include a combination of TMB and immune signatures. Modulation of TME may be a reasonable therapeutic strategy in order to orchestrate genomic and immunophenotypic status. It is still to be determined whether CGP panels for clinical specimens should be expanded to evaluate immune signatures in addition to genomic analysis of tumor-intrinsic alterations. Integration of RNA- and DNA-sequencing is emerging; the addition of immuno-oncology analyses in assays such as Tempus $\mathrm{xT}$ (including TMB, PD-1/PDL1 expression, microsatellite instability, and research-use only gene expression analysis of the TME and neoantigen prediction) may help to clarify this question (25). The role of TMB and TME in identifying patient groups who may benefit from immunotherapy should be determined in future randomized control trials. Combination strategies may ultimately emerge to convert high TMB non-T cellinflamed tumors into $\mathrm{T}$ cell-inflamed tumors as a means to sensitize tumors to therapies dependent on T-cell killing.

\section{Acknowledgments}

The authors thank Dr. Ronald DeLellis for his review of the manuscript.

Funding: None.

\section{Footnote}

Conflicts of Interest: The authors have no conflicts of interest to declare.

Ethical Statement: The authors are accountable for all aspects of the work in ensuring that questions related to the accuracy or integrity of any part of the work are appropriately investigated and resolved.

Open Access Statement: This is an Open Access article distributed in accordance with the Creative Commons Attribution-NonCommercial-NoDerivs 4.0 International License (CC BY-NC-ND 4.0), which permits the noncommercial replication and distribution of the article with the strict proviso that no changes or edits are made and the original work is properly cited (including links to both the formal publication through the relevant DOI and the license). See: https://creativecommons.org/licenses/by-nc-nd/4.0/.

\section{References}

1. Aggen DH, Drake CG, Rini BI. Targeting PD-1 or PDL1 in Metastatic Kidney Cancer - Combination Therapy in the First Line Setting. Clin Cancer Res 2020. [Epub ahead of print]. 
2. Zhang C, Li Z, Qi F, et al. Exploration of the relationships between tumor mutation burden with immune infiltrates in clear cell renal cell carcinoma. Ann Transl Med 2019;7:648.

3. Alexandrov LB, Nik-Zainal S, Wedge DC, et al. Signatures of mutational processes in human cancer. Nature 2013;500:415-21.

4. Chalmers ZR, Connelly CF, Fabrizio D, et al. Analysis of 100,000 human cancer genomes reveals the landscape of tumor mutational burden. Genome Med 2017;9:34.

5. Lawrence MS, Stojanov P, Polak P, et al. Mutational heterogeneity in cancer and the search for new cancerassociated genes. Nature 2013;499:214-8.

6. Turajlic S, Litchfield K, Xu H, et al. Insertion-anddeletion-derived tumour-specific neoantigens and the immunogenic phenotype: a pan-cancer analysis. Lancet Oncol 2017;18:1009-21.

7. McDermott DF, Huseni MA, Atkins MB, et al. Clinical activity and molecular correlates of response to atezolizumab alone or in combination with bevacizumab versus sunitinib in renal cell carcinoma. Nat Med 2018;24:749-57.

8. Rooney MS, Shukla SA, Wu CJ, et al. Molecular and genetic properties of tumors associated with local immune cytolytic activity. Cell 2015;160:48-61.

9. Cherkasova E, Malinzak E, Rao S, et al. Inactivation of the von Hippel-Lindau tumor suppressor leads to selective expression of a human endogenous retrovirus in kidney cancer. Oncogene 2011;30:4697-706.

10. Thorsson V, Gibbs DL, Brown SD, et al. The Immune Landscape of Cancer. Immunity 2018;48:812-30.

11. Yarchoan M, Hopkins A, Jaffee EM. Tumor Mutational Burden and Response Rate to PD-1 Inhibition. N Engl J Med 2017;377:2500-1.

12. Samstein RM, Lee CH, Shoushtari AN, et al. Tumor mutational load predicts survival after immunotherapy across multiple cancer types. Nat Genet 2019;51:202-6.

13. Goodman AM, Kato S, Bazhenova L, et al. Tumor mutational burden as an independent predictor of response to immunotherapy in diverse cancers. Mol Cancer Ther 2017;16:2598-608.

14. McGranahan N, Furness AJ, Rosenthal R et al. Clonal neoantigens elicit $\mathrm{T}$ cell immunoreactivity and sensitivity to immune checkpoint blockade. Science 2016;351:1463-9.

15. Maleki Vareki S. High and low mutational burden tumors versus immunologically hot and cold tumors and response to immune checkpoint inhibitors. J Immunother Cancer 2018;6:157.

16. Luke JJ, Bao R, Sweis RF, et al. WNT/ $\beta$-catenin Pathway Activation Correlates with Immune Exclusion across Human Cancers. Clin Cancer Res 2019;25:3074-83.

17. Fridman WH, Pagès F, Sautès-Fridman C, et al. The immune contexture in human tumours: impact on clinical outcome. Nat Rev Cancer 2012;12:298-306.

18. Nakano O, Sato M, Naito Y, et al. Proliferative activity of intratumoral CD8(+) T-lymphocytes as a prognostic factor in human renal cell carcinoma: clinicopathologic demonstration of antitumor immunity. Cancer Res 2001;61:5132-6.

19. Giraldo NA, Becht E, Pagès F, et al. Orchestration and Prognostic Significance of Immune Checkpoints in the Microenvironment of Primary and Metastatic Renal Cell Cancer. Clin Cancer Res 2015;21:3031-40.

20. Iglesia MD, Parker JS, Hoadley KA, et al. Genomic Analysis of Immune Cell Infiltrates Across 11 Tumor Types. J Natl Cancer Inst 2016. doi: 10.1093/jnci/djw144.

21. Chen B, Khodadoust MS, Liu CL, et al. Profiling Tumor Infiltrating Immune Cells with CIBERSORT. Methods Mol Biol 2018;1711:243-59.

22. Wang X, Li M. Correlate tumor mutation burden with immune signatures in human cancers. BMC Immunol 2019;20:4.

23. Ribas A, Dummer R, Puzanov I, et al. Oncolytic Virotherapy Promotes Intratumoral T Cell Infiltration and Improves Anti-PD-1 Immunotherapy. Cell 2017;170:1109-19.

24. Loi S, Dushyanthen S, Beavis PA, et al. RAS/MAPK Activation Is Associated with Reduced Tumor-Infiltrating Lymphocytes in Triple-Negative Breast Cancer: Therapeutic Cooperation Between MEK and PD-1/PDL1 Immune Checkpoint Inhibitors. Clin Cancer Res 2016;22:1499-509.

25. Beaubier N, Bontrager M, Huether R, et al. Integrated genomic profiling expands clinical options for patients with cancer. Nat Biotechnol 2019;37:1351-60.
Cite this article as: Yakirevich E, Patel NR. Tumor mutational burden and immune signatures interplay in renal cell carcinoma. Ann Transl Med 2020;8(6):269. doi: 10.21037/atm.2020.02.81 